\title{
3D Folded Loop UAV Antenna Design
}

\author{
Alexander Pyattaev ${ }^{1}$, Dmitri Solomitckii ${ }^{2}$, and Aleksandr Ometov ${ }^{2}$ \\ ${ }^{1}$ Peoples Friendship University of Russia (RUDN University), Russia \\ 2 Tampere University of Technology, Tampere, Finland \\ Email: aleksandr.ometov@tut.fi \\ Abstract. Utilization of Unmanned Aerial Vehicles (UAVs), also know \\ as "drones" has a great potential for many emerging applications, such \\ as delivering the connectivity on-demand, providing services for public \\ safety, or recovering after damage to the communication infrastructure. \\ Notably, nearly any application of drones requires a stable link to the \\ ground control center, yet this functionality is commonly added at the \\ last moment in the design, necessitating compact antenna designs. In \\ this work, we propose a novel electrically small antenna element based \\ on the 3D folded loop topology, which could be easily located inside \\ the UAV airframe, yet still delivering good isolation from the drones \\ own noise sources. The complete manufacturing technique along with \\ corresponding simulations/measurements are presented. Measurements \\ and evaluations show that the proposed antenna design is an option to \\ achieve genuinely isotropic radiation in a small size without sacrificing \\ efficiency.
}

\section{Introduction}

Unmanned Aerial Vehicles (UAVs) are viral today in a variety of applications, ranging from civilian entertainment all the way to the military use [1-4]. In all of those cases, it is critical for the UAV to keep the telemetry/control link with its operator at all time (in case the flight is not fully autonomous), as to ensure that the UAV remains in controlled flight [5].

In addition, for certain applications of drones reliable communication is the whole point of operation (even if the drone itself is automated). For instance, an area may be left without any connectivity due to the natural disaster [6]. To address this, a cell relay [7] or a stand-alone base station (BS) [8] may be considered. However, this is always very costly to do [9], and thus the use of UAVs [10] as relays may be attractive.

The primary challenge of any ground-to-air comms could be formalized as that most of the antennas utilized today are not isotropic, and thus their gain may vary depending on the orientation of the UAV relative to the operator [11]. Preferably, a UAV should be equipped with an isotropic antenna, as to ensure predictable levels of radio signal irrespective of the orientation. In addition, the number of antennas used should be minimal to save weight.

Of course, merely placing an isotropic antenna onto the UAV does not solve the problem, since the body of the aircraft is not entirely transparent to radio signals, and thus will block the signal depending on the position of the antenna. Due to small sizes of the typical UAV's compared to larger aviation, the design requirements for the antenna become very strict [12], as it also has to fit inside the UAV body for best aerodynamic performance. 
The primary goal of this work was to design an antenna system for a fixedwing UAV that would ensure uniform illumination around the airplane, while at the same time minimally interfering with the other aspects of UAV design. The results, however, are equally applicable to quadrotors and ground vehicles.

The remainder of the manuscript is as follows. First, we explain the key constraints in more details in Section 2. Section 3 provides the design process, discussion and simulation results. Then, we continue to the actual manufacturing process description and measurement results in Section 4. The last section concludes the manuscript.

\section{Antenna requirements}

As stated before, the goal of this work is to develop an antenna system for a UAV. We aimed at the airplane structure built with a conventional aerodynamic scheme in mind (single straight wing with classic tail assembly), keeping in mind that similar structure should be applicable to rotorcraft as well. The schematic drawing of the airframe is given in the Fig. 1.

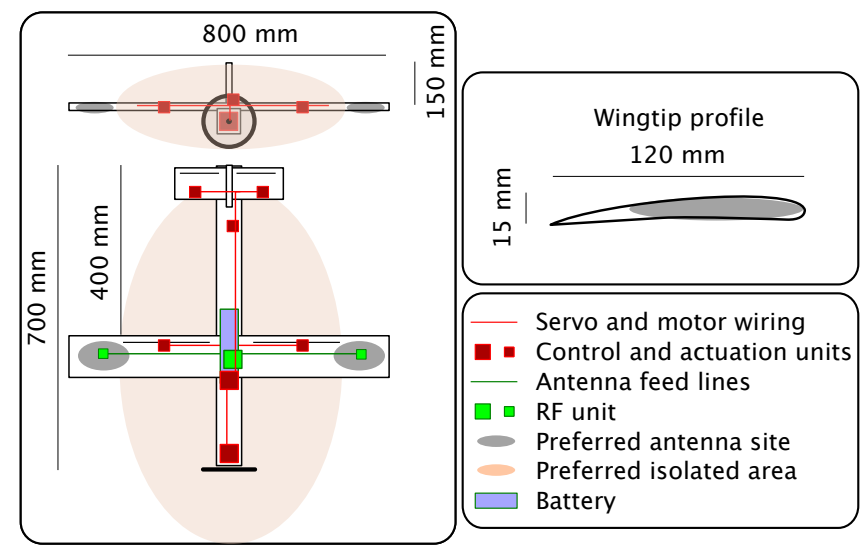

Fig. 1. Selected UAV schematics.

The key requirements chosen for the developed system are summarized as follows:

- Isotropic radiation pattern in the far-field around the airplane;

- At least $-20 \mathrm{~dB}$ isolation from the electrics inside the airplane body;

- Mounting inside the airfoils of the airplane (no parts of antenna sticking outside of the plane);

- Small size and weight (wire antenna preferred to horns and patches);

- Single operating frequency around $2.5 \mathrm{GHz}$;

- At least $95 \%$ radiation efficiency (for maximal coverage).

The requirements listed before are based on the previous experience making UAV's for entertainment purposes, and problems observed with RF interferences as a result thereof. Further, we referred to the commercial examples of UAV antenna design [13]. 
As discussed above, the isotropic radiation pattern is key to the sustained, reliable control of the airplane. The isolation requirement stems from the fact that most of the avionic controls are brushed electric motors, which are known for massive commutation noise levels. Similarly, control units employ Pulse-width modulation (PWM) techniques to change the power supplied to the primary motors, which, in turn, use MOSFET switches, all of them creating high-frequency noise harmonics.

Further, large lengths of wires and metallic structural components such as landing gear in the airplane could interfere with the operation of the antennas if not taken care of properly, while carbon fiber elements are excellent RF absorbers at certain frequencies. Therefore, it is highly preferred to isolate the airplane from the antenna altogether, as to simplify its design. As a direct consequence of these requirements, we may easily identify the locations best suited for the antenna placement. Those are located in the wing tips and are highlighted in gray in Fig. 1. The wingtips are far away from most motors, and are usually composed of plastic (and are thus transparent to the radio waves of the selected frequency). Furthermore, they are mostly empty inside since they have very low structural load. In what follows, we will use the wingtip geometry (see Fig. 1) as our guideline for the size limits on the antenna elements. Mainly, we are limited by the wingtip height being about $15 \mathrm{~mm}$ for most consumer-grade UAVs.

One should note that in commercial airplanes it is commonly preferred to position the antenna outside of the fuselage since the body is made of aluminum and is not RF-transparent. Further, the relatively small antenna elements do not cause significant drag compared to the plane itself. In small gliders and UAV's, however, even thin wire antennas cause significant aerodynamic drag and thus should be positioned inside the body of the airplane.

Based on the proposed use-case, the antenna system should provide uniform coverage in a horizontal plane ( $3 \mathrm{~dB}$ fluctuations are $\mathrm{OK})$, and near-uniform coverage in a vertical plane ( $5 \mathrm{~dB}$ fluctuations are $\mathrm{OK})$. Next, based on the geometry of our example aircraft, the antenna elements must be located in areas $120 \times 15 \times 200 \mathrm{~mm}$ in size symmetrically on the tips of the airplane wings. The spacing between antennas is approximately $800 \mathrm{~mm}$.

From the efficiency and cost point of view, the Standing wave ratio (SWR) for the antenna must not exceed $-20 \mathrm{~dB}$; antenna should be matched to $50 \mathrm{Ohm}$ for coupling with standard radios.

\section{Antenna design - 3D folded loop}

The simplest wire antenna - a dipole - does not provide the desired performance due to its high vertical size (much more than allowed $15 \mathrm{~mm}$ ), inappropriate coverage pattern and potentially high coupling to the airplane electrics. However, two dipoles separated by $\lambda / 4$ can produce a cardioid pattern, and its null can be used to negate the coupling with airplane electrics, thus addressing most of the requirements. The only questions then become a) how to make the dipoles fit into the wing's $15 \mathrm{~mm}$ height without losing efficiency, and b) how to address the lack of coverage strictly downwards, which could be important for some applications. 
The most natural alternative to a conventional dipole is the loop antenna. Unlike comparable dipole, it better conforms with the shape of the wing. Controversially, the diameter of the loop ends up being so large $(c / 2500 \mathrm{MHz} / p i \approx 38 \mathrm{~mm})$, that the two loops needed to produce a cardioid pattern $(c / 2500 \mathrm{MHz} / 4 \approx 30$ $\mathrm{mm}$ ) overlap with each other. Of course, there is a possibility to bend the loop antenna into a more "rectangular" shape without changing its radiation pattern too much, but unfortunately, this results in mutual coupling between loops, and the desired array pattern is not achieved.

Faced with the problem of making a loop antenna even smaller, one has two paths that can be followed: (i) To place the loop on dielectric substrate as to reduce its size (sacrificing efficiency); (ii) To fold the loop even further to make it electrically small.

Since the dielectric substrate option inevitably causes loss of efficiency, we have taken the challenge to follow the second path, and design an array element that would be small enough to fit into a wing and stay isolated from the other element, yet at the same time be efficient as a full-size antenna. The antenna we have used is commonly known as 3D folded loop (3DFL) structure.

In practical engineering, 3DFLs are sometimes used by amateur radio enthusiasts to make small-size, long-wavelength antenna due to the simplicity of manufacturing and modeling with NEC-like tools [14]. Academically, however, the $3 \mathrm{DFL}$ theory is very poorly developed in the literature (the only example is found in work [15]), and thus does not find wide commercial use. However, it is straightforward to explain the fundamental principles behind 3DFL antennas.

3DFL antenna is a 3D hybrid structure combining loop and meander antenna principles, and consists of at least three components:

- The first radiating loop, which is smaller than the full wavelength and would normally radiate at a very high frequency. This loop is fed in the middle and matched to $50 \mathrm{Ohm}$ feed line;

- The connecting structure, which acts as transmission line and delays the wave, thus effectively enlarging the antenna;

- The second radiating loop, which complements the first one and balances the entire structure. The second loop may be oriented almost arbitrarily with respect to the first one, but best characteristics are usually achieved when it is coplanar to the first one.

Overall, the full wire length of the 3DFL antenna is almost equal to the resonant wavelength, and electrically it acts as a resonant, free-space antenna. Externally, however, it ends up being smaller than its dipole or loop counterparts.

The design starts with a conventional, resonant loop antenna. It is clear that there are two active zones based on the current intensity distribution, as shown in Fig. 2, and two regions which barely have any current flow at all. Those can be folded and bent in any way desired with minimal effects on the current distribution, antenna pattern or efficiency. The amount of bending that can be done with minimal impact is quite generous, and the optimal amount is a subject of separate research. 

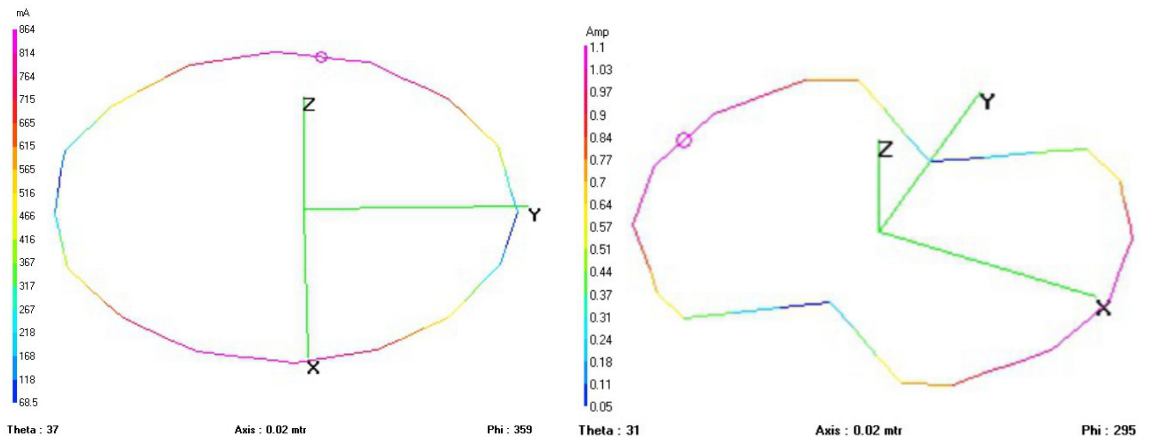

Fig. 2. Conventional (left) and bent (right) loop antenna current distributions.

$$
\begin{array}{|l}
m< \\
959 \\
904 \\
849 \\
794 \\
739 \\
684 \\
629 \\
574 \\
519 \\
464 \\
409 \\
354 \\
299 \\
2944 \\
189 \\
135 \\
7996 \\
\hline
\end{array}
$$
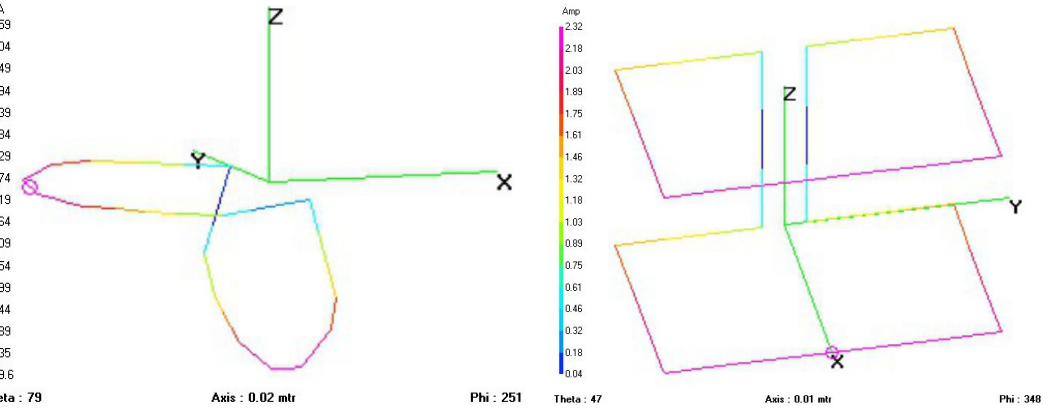

Fig. 3. 3DFL transition: bending off-plane (left) and completed 3DFL (right).

The logical next step is to bend the antenna off its plane, transitioning from flat into the 3D structure, as it is shown in Fig. 4. This allows to further work towards a truly 3D structure, as can be seen in Fig. 3. As before, the pattern and operating frequency are almost unaffected by those manipulations as long as the antenna perimeter (i.e., the full length of all wires) remains constant, and bending occurs at the points with minimal current density. As there is no clear way to analytically model 3DFL structures, through the bending process, one must constantly check the resonant frequency of the resulting antenna, and adjust its structure as needed, trying to meet the dimensional constraints of the design. Clearly, for isotropic pattern one must minimize the bounding volume of the resulting structure.

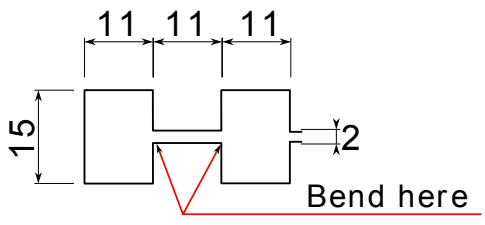

Fig. 4. Antenna bending and dimensions. 
Note, that 3D bending design can produce a structure which is essentially a cube, and thus has the maximal surface area with minimal volume, which is the desired effect. In fact, the completed 3DFL antenna has almost three times smaller side than equivalent planar structure, occupying just $11 \times 11 \times 15 \mathrm{~mm}$ volume (see Fig. 4), compared to planar loop's $38 \mathrm{~mm}$ diameter. As a result, resonant 3DFL structure has nearly isotropic pattern (since it is so small), and can be packed much tighter to other element, including desired $\lambda / 4$ spacing needed to produce cardioid pattern.

On the other hand, just like full-size loop structures, they have reasonably good resonant characteristics and are very robust to near-field obstructions. Further, due to the feed positioning on the bottom of the structure, it is easy to run the feed lines for this sort of antennas when used in array.
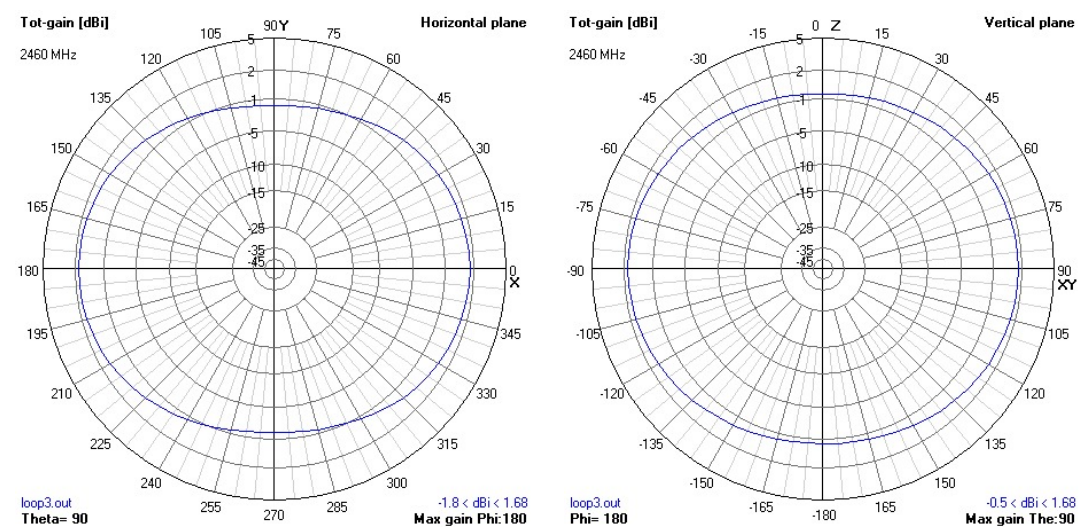

Fig. 5. Radiation pattern for 3DFL: horizontal (left) and vertical (right) patterns.

The resulting 3DFL structure, even though it is resonant, does have several curious properties. First and most important one is that it is essentially a perfect isotropic radiator. As can be seen from Fig. 5, the pattern is indeed uniform in every direction within $1 \mathrm{~dB}(!)$ tolerance. There are no hidden nulls/cavities in this pattern, and it is only natural that this antenna radiates in such a way. Since its radiating parts are so much shorter than $\lambda$, in fact as short as $\lambda / 8$, it is as an electrically small antenna and thus has near isotropic pattern. On the other hand, just like a full-size resonant antenna, it has near about $99 \%$ theoretical radiation efficiency, since no dielectric was used to reduce its size.

On its own, the 3DFL antenna is not well-matched to $50 \mathrm{Ohms}$ and requires external lumped component matching network. However, the typical component values are minimal ( $5 \mathrm{pF}$ capacitors and 5 nHenry inductors) and present no significant engineering issue. With the lumped component matching network in place, 3DFL antenna shows outstanding performance at operating frequency, as shown in Fig. 6.

Array matching of 3DFL antennas is a somewhat tedious process, as they couple to each other and there is a need to perform matching after the array is 

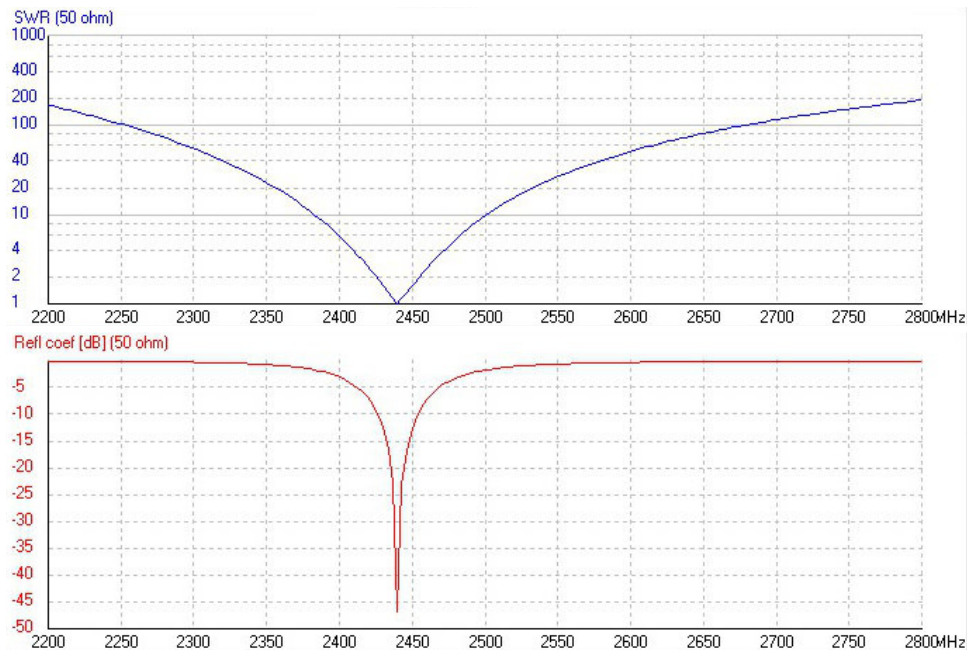

Fig. 6. Radiation pattern for 3DFL structure.

constructed. In addition, at this stage one needs to balance the impedances and ensure correct power distribution between elements (otherwise one element tends to radiate more energy, harming the array performance). However, resulting system shows good performance within our test airplane (see Fig. 7 for crucial performance data).

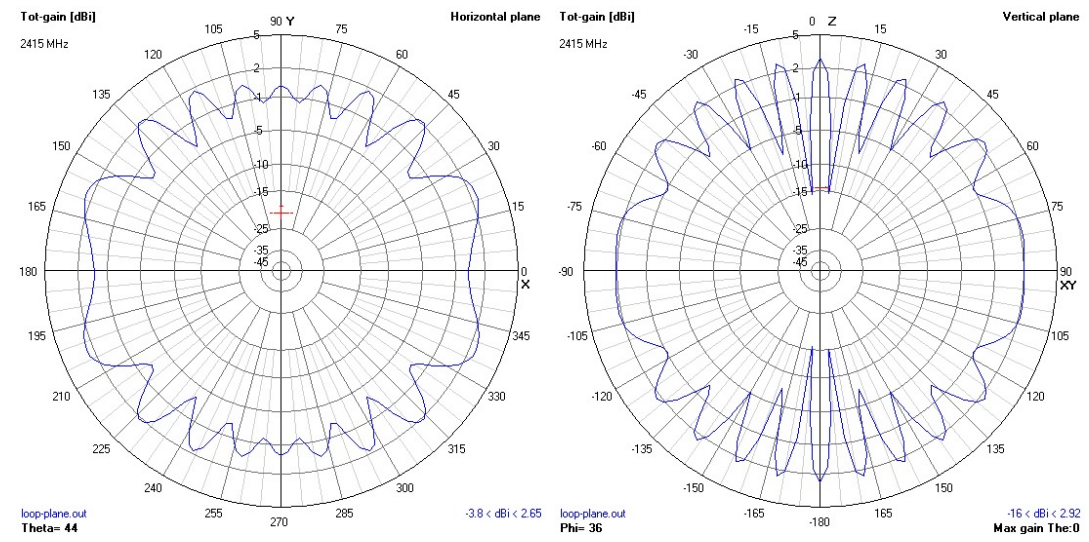

Fig. 7. Simulations of the finalized antenna array: horizontal (left); vertical (right).

Electrically, the resulting antenna array is a narrowband (about $1 \%$ fractional bandwidth), resonant structure with high efficiency. Even though the matching would hold over a larger bandwidth, the array can only be operated with narrowband signals due to element spacing and phases of the feeds, as shown in Fig. 8.

Another interesting observation may be found in Fig. 9. Here, when operating under incorrect frequency, the antenna pattern of individual sub-arrays turns into quasi-omnidirectional, with a spectacular set of grating lobes. Therefore, even though antenna elements do radiate fine under different frequencies, their use within arrays must be targeted at one specific frequency. However, 2 in- 


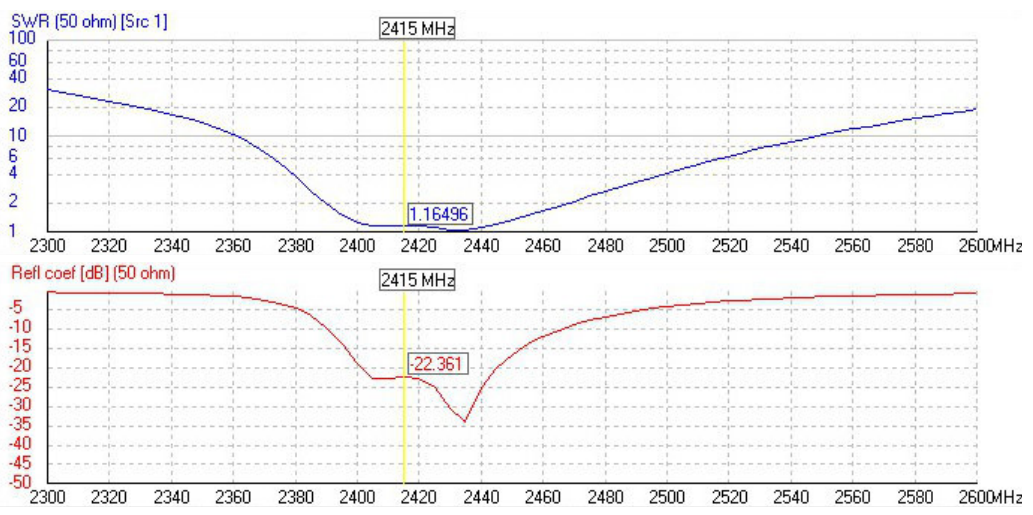

Fig. 8. Matching characteristics of the completed array.

dividual elements may be used if motor noise isolation is not required to provide isotropic coverage.

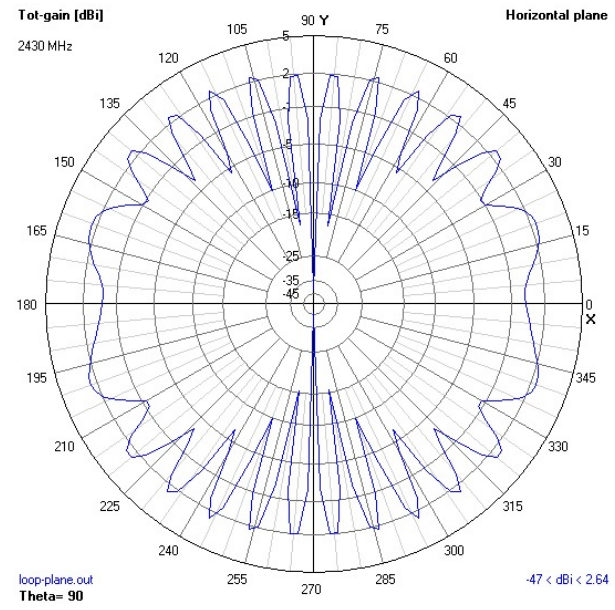

Fig. 9. Off-frequency array operation $(+35 \mathrm{MHz})$.

\section{Measurements and resulting antenna element specification}

\subsection{Post-manufacturing}

Since the development of the entire array structure with equipment avaialbe proved to be a complex task, we have decided to evaluate a single antenna element as to prove the feasibility of the design. Single elements of this antenna require matching after manufacture and can be tuned in the wide range of frequencies. In this example, we have tuned a testing antenna to operate at 2.5 $\mathrm{GHz}$ (see Fig. 10).

The practical antenna appears to be insensitive to interfering metal objects at distances above $3 \mathrm{~cm}$. Thus, it is a very robust antenna for applications requiring small footprint with a high number of obstacles around. Such tight near field 

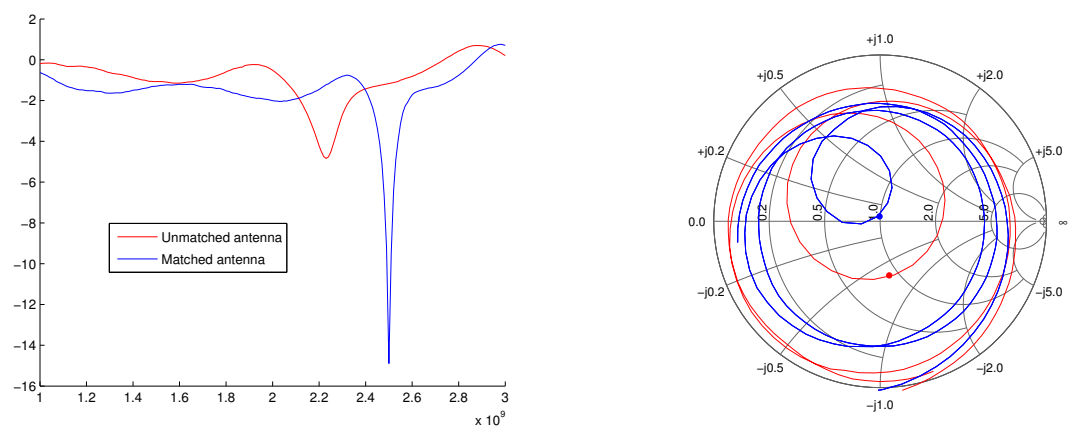

Fig. 10. Measured characteristics of the manufactured antenna before/after matching.

also means that this antenna is ideally suited for the near-field measurement applications. The manufactured and matched antenna was taken to SATIMO $^{1}$ StarLab measurement site, and its field pattern was thoroughly measured.

\subsection{Radiation pattern measurements}

In the far field, a single element should have (according to the simulations) an almost perfectly isotropic pattern. Thus, we have thoroughly tested that the manufactured element conforms to this requirement. One of the main concerns was the effect of the feed point, where the coaxial cable could interfere with the antenna radiation. Since SATIMO equipment does not allow for full 360degree vertical sweep, the radiation pattern was measured with two different orientations of the antenna, and two different antennas, as shown in Fig. 11.
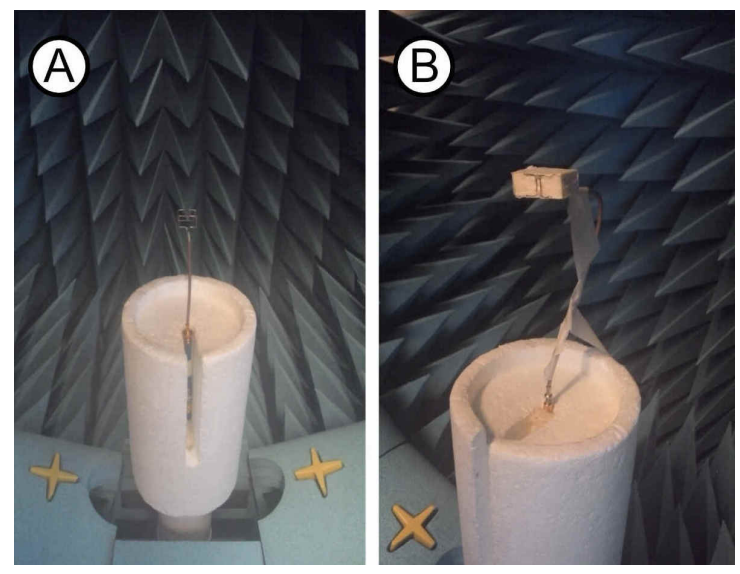

Fig. 11. Radiation field measurement setups.

The two antennas have been both tuned to the same frequency, and oriented such that the feed point loop is oriented vertically in the $\mathrm{XZ}$ plane (with the

\footnotetext{
${ }^{1}$ Microwave Vision Group: StarLab: http://www.mvg-world.com/en/products/ field_product_family/antenna-measurement-2/starlab
} 

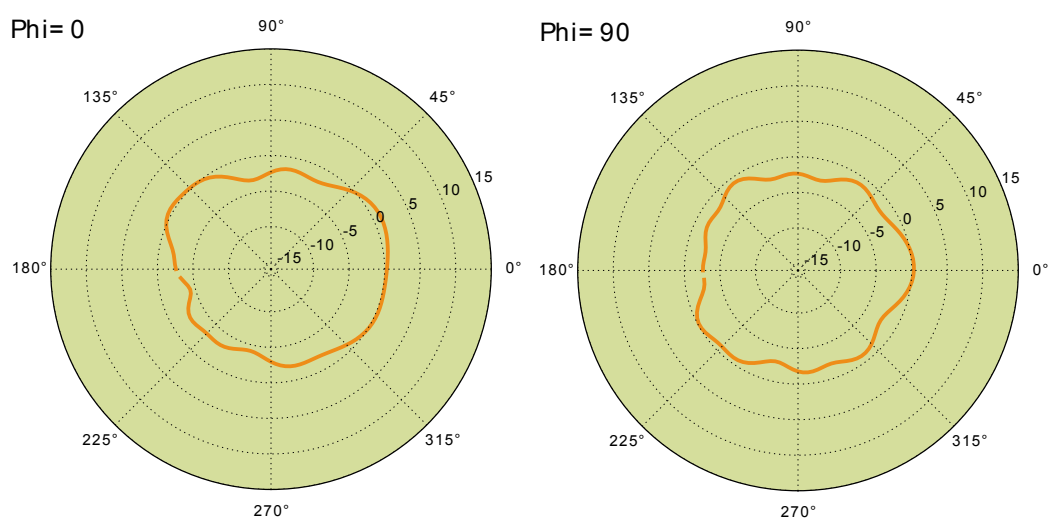

Fig. 12. Measured radiation pattern, vertical slices
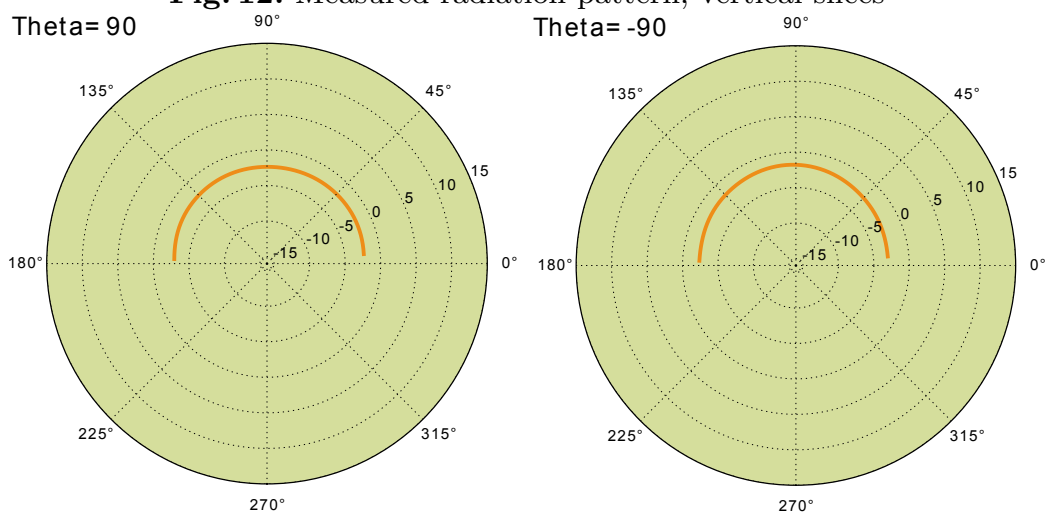

Fig. 13. Measured radiation pattern, horizontal slices

feed coming from negative $\mathrm{Z}$ direction) or horizontally in the XY plane (with the feed coming from positive $\mathrm{Y}$ direction). Since their patterns are omnidirectional, the precise alignment of the orientation was not necessary.

To better numerically see how the pattern differs for various mount orientations, one can refer to Figs. 12 and 13. The scale used here is $-15: 15 \mathrm{~dB}$, to more clearly see how uniform the radiation pattern is. While making measurements of such antennas, one must take care to always use the right scaling on all axes, as most software tends to over-emphasize the irregularities in the pattern.

The antenna element radiation pattern is nearly isotropic. The isotropic property holds in both orientations (A and B) and with changing the measured antenna element. The minimal observed gain has been $-2.5 \mathrm{~dB}$, the maximal observed gain was $+2.0 \mathrm{~dB}$. We believe that the final element design well meets its design specifications, despite limitations of manufacturing facilities. 


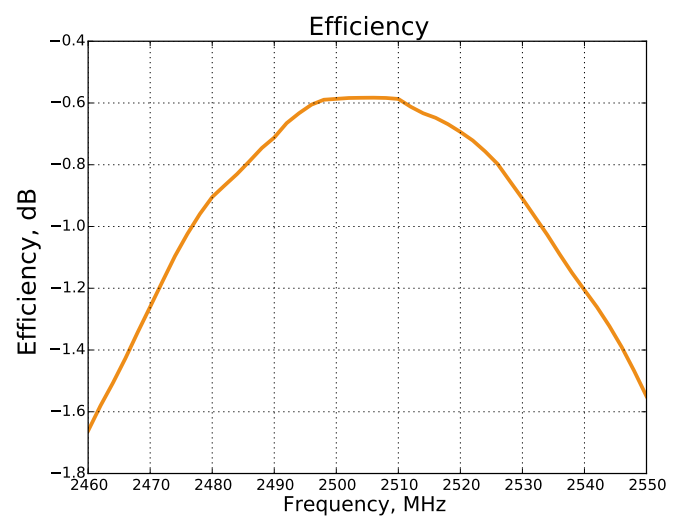

Fig. 14. Radiation efficiency

Finally, the efficiency of the antenna is, as expected, very good at the operating frequency, which can be seen from Fig. 14. Numerically, the efficiency is about $90 \%$ for all produced antenna elements.

\section{Conclusions}

Today, the analysis of the drone applications is a trend in both industrial and academic communities. Currently utilized antennas to maintain the drone flight may be affected by the interference delivered by the UAV itself, as well as by undesired nulls towards ground control site. In this manuscript, we have designed a complete antenna system for a drone. The design has the following key features: (i) allows the UAV to communicate with targets in all directions with minimal gain fluctuations; (ii) isolates the antennas from electrics in the plane itself, avoiding self-interference; (iii) allows deployment completely inside the airplane body; (iv) completed antenna is very lightweight ( 2 grams with foam support structure).

The antenna system uses a novel type of element, 3D folded loop, which is a state of the art antenna structure, only recently becoming popular. The manufacturing technique was also developed to produce the 3DFL antennas in the lab environment with minimal equipment available. Singular elements have been successfully produced and tested, confirming their unique properties (minimal size of $\lambda / 10$ with $90 \%$ measured radiation efficiency, isotropic pattern). This manuscript delivers a complete set of instructions to produce the 3DFL antenna along with its properties.

Measurements and evaluations show that 3DFL is a relatively easy way to achieve truly isotropic radiation in a small size without sacrificing efficiency. We believe that 3DFL-based antennas will often be used for UAVs and other size-weight-critical systems. However, we would not recommend producing such structures without appropriate equipment, such as metalization 3D printers.

\section{Acknowledgment}

The publication has been prepared with the support of the "RUDN University Program 5-100". 


\section{References}

1. Bor-Yaliniz, I., Szyszkowicz, S.S., Yanikomeroglu, H.: Environment-Aware DroneBase-Station Placements in Modern Metropolitans. IEEE Wireless Communications Letters (2017)

2. Orsino, A., Ometov, A., Fodor, G., Moltchanov, D., Militano, L., Andreev, S., Yilmaz, O.N., Tirronen, T., Torsner, J., Araniti, G., et al.: Effects of Heterogeneous Mobility on D2D-and Drone-Assisted Mission-Critical MTC in 5G. IEEE Communications Magazine 55(2) (2017) 79-87

3. Paramonov, A., Nurilloev, I., Koucheryavy, A.: Provision of Connectivity for (Heterogeneous) Self-organizing Network Using UAVs. In: Internet of Things, Smart Spaces, and Next Generation Networks and Systems. Springer (2017) 569-576

4. Mäkitalo, N., Ometov, A., Kannisto, J., Andreev, S., Koucheryavy, Y., Mikkonen, T.: Safe and Secure Execution at the Network Edge: A Framework for Coordinating Cloud, Fog, and Edge. IEEE Software (2018)

5. Secinti, G., Darian, P.B., Canberk, B., Chowdhury, K.R.: SDNs in the Sky: Robust End-to-End Connectivity for Aerial Vehicular Networks. IEEE Communications Magazine 56(1) (2018) 16-21

6. Ometov, A., Sopin, E., Gudkova, I., Andreev, S., Gaidamaka, Y.V., Koucheryavy, Y.: Modeling Unreliable Operation of mmWave-Based Data Sessions in MissionCritical PPDR Services. IEEE Access 5 (2017) 20536-20544

7. Müller, W., Marques, H., Pereira, L., Rodriguez, J., Brouwer, F., Bouwers, B., Politis, I., Lykourgiotis, A., Ladas, A., Adigun, O., et al.: Secure and interoperable communication infrastructures for PPDR organisations. In: SPIE Defense+ Security, International Society for Optics and Photonics (2016)

8. Deruyck, M., Wyckmans, J., Martens, L., Joseph, W.: Emergency ad-hoc networks by using drone mounted base stations for a disaster scenario. In: Proc. of 12th International Conference on Wireless and Mobile Computing, Networking and Communications (WiMob), IEEE (2016) 1-7

9. ICS Industries PTY LTD: Cell on Wheels: Engineered for All Environments. ([online]. Available: http://www.icsindustries.com.au/downloads/brochures?download=37:cell-onwheels, 2017.)

10. Solomitckii, D., Gapeyenko, M., Semkin, V., Andreev, S., Koucheryavy, Y.: Technologies for Efficient Amateur Drone Detection in 5G Millimeter-Wave Cellular Infrastructure. IEEE Communications Magazine 56(1) (2018) 43-50

11. Fabra, F., Calafate, C.T., Cano, J.C., Manzoni, P.: On the impact of inter-UAV communications interference in the $2.4 \mathrm{GHz}$ band. In: Proc. of 13th International Wireless Communications and Mobile Computing Conference (IWCMC), IEEE (2017) 945-950

12. Moses, A., Rutherford, M.J., Valavanis, K.P.: Radar-based detection and identification for miniature air vehicles. In: Proc. of IEEE International Conference on Control Applications (CCA), IEEE (2011) 933-940

13. Cobham Antenna Systems: Cobham Antenna Systems. Unmanned Systems Antennas Airborne Platforms, UAVs, Ground Vehicles, Robots. Technical report, Technical Report (2010)

14. Cuthbert, D.: The 3-D Folded Loop Antenna. Technical report, Technical Report (2003)

15. Chiu, C.W., Chang, C.H., Chi, Y.J.: A compact folded loop antenna for LTE/GSM band mobile phone applications. In: Proc. of International Conference on Electromagnetics in Advanced Applications (ICEAA), IEEE (2010) 382-385 